\title{
In vivo antitumor activity evaluation of cancer vaccines prepared by various antigen forms in a murine hepatocellular carcinoma model
}

\author{
CHUNFENG SI ${ }^{1 *}$, MAOLEI XU ${ }^{1 *}$, MEIYU LU ${ }^{1}$, YAN YU $^{2}$, MEIZI YANG ${ }^{1}$, \\ MIAOMIAO YAN ${ }^{1}$, LING ZHOU ${ }^{1}$ and XIAOPING YANG ${ }^{1}$
}

\begin{abstract}
${ }^{1}$ Key Laboratory of Traditional Chinese Medicine Prescription Effect and Clinical Evaluation of State Administration of Traditional Chinese Medicine, School of Pharmacy; ${ }^{2}$ Medicine and Pharmacy Research Center, Binzhou Medical University, Yantai, Shandong 264003, P.R. China
\end{abstract}

Received January 20, 2016; Accepted October 3, 2017

DOI: $10.3892 /$ ol.2017.7169

\begin{abstract}
Cancer cell vaccines with strong specificity and low tolerance have been revealed to be a promising option for oncology treatment. Various antigen forms, including tumor cell lysate and glutaraldehyde-fixed tumor cells, have been intensively used in cancer vaccine preparation. However, the most effective antigen form has not yet been identified. In the present study, the antitumor efficiency of vaccines prepared by these two antigen forms was systematically investigated. Murine H22 hepatocellular carcinoma cell lysate and glutaraldehyde-fixed $\mathrm{H} 22$ hepatocellular carcinoma cells were conjugated with Freund's adjuvant to prepare vaccines, H22-TCL and Fixed-H22-CELL, respectively. H22-TCL and Fixed-H22-CELL were administrated by subcutaneous immunization in prophylactic and therapeutic strategies. The results of the present study revealed that H22-TCL immunization induced more significant inhibition on tumor growth and metastasis compared with Fixed-H22-CELL injection. Furthermore, histopathological observation demonstrated that H22-TCL vaccine induced larger areas of continuous necrosis within tumors compared to the Fixed-H22-CELL vaccine, which was associated with the extent of tumor inhibition. More importantly, the H22-TCL vaccine injection elicited more evident antigen-specific antibody responses compared with the Fixed-H22-CELL injection. Splenocytes from H22-TCL vaccinated mice also exhibited a more
\end{abstract}

Correspondence to: Dr Xiaoping Yang or Dr Ling Zhou, Key Laboratory of Traditional Chinese Medicine Prescription Effect and Clinical Evaluation of State Administration of Traditional Chinese Medicine, School of Pharmacy, Binzhou Medical University, 346 Guanhai Road, Yantai, Shandong 264003, P.R. China

E-mail: yangxiaoping@bzmc.edu.cn

E-mail: zhouling-2007-ok@163.com

"Contributed equally

Key words: cancer immunotherapy, tumor cell lysate, glutaraldehydefixed tumor cell, hepatocellular carcinoma significant $\mathrm{T}$ lymphocytes proliferation compared with that from Fixed-H22-CELL-treated mice. All the results indicated that whole tumor cell lysate may be a more effective antigen form in cancer vaccine preparation compared with glutaraldehyde-fixed tumor cells, which elicited more marked antigen specific humoral and cellular immune responses resulted with a superior antitumor efficiency. This would have important clinical signification for cancer vaccine preparation and serve a role in prompting this to other researchers.

\section{Introduction}

Immunotherapy has become an important alternative option in oncology treatment, which represents a promising method for patients due to its specificity for tumor cells and sustained immunological memory that may safeguard against recurrences (1). Application of tumor vaccines to elicit antigen specific immune responses for cancer treatment is a hot area of immunotherapy research. Inactivated whole tumor cells providing the total array of antigens expressed by the individual tumor are the most commonly utilized antigen types in tumor vaccine research (2-4).

Currently, the known vaccine types utilized as whole tumor cells include irradiated tumor cells, glutaraldehyde-fixed tumor cells and tumor cell lysates (5-9). In particular, along with the development of tumor adjuvant, the efficiency of whole tumor cell vaccines has been markedly improved. In a previous study, a whole hepatocellular carcinoma cell lysate-based vaccine with diphtheria toxin and two tandem repeats of mycobacterial HSP70 fragment 407-426 $\left(\mathrm{M}_{2}\right)$ as adjuvant exhibited modest antitumor effects in the preventive procedure $(10,11)$.

Although whole tumor cell vaccines have already been widely studied, vaccine therapy has yielded suboptimal clinical results in therapeutic procedures $(12,13)$. Furthermore, it is not clear which antigen form is more effective in cancer vaccine preparation. Therefore in the present study, tumor cell lysate (TCL) and glutaraldehyde-fixed tumor cells, two commonly used antigen forms, were investigated to improve the present vaccine strategy. Murine H22 hepatocellular carcinoma cell lysate and glutaraldehyde-fixed $\mathrm{H} 22$ hepatocellular carcinoma 
cells were conjugated with Freund's adjuvant to prepare vaccines, H22-TCL and Fixed-H22-CELL, respectively. The antitumor efficacy of these two vaccines was evaluated using a subcutaneous hepatocellular carcinoma and an experimental metastasis model. Subsequently, the elicited H22-specific antibodies were detected using the enzyme-linked immunosorbent assay (ELISA) method. An MTT assay was performed to determine the proliferation ability of lymphocytes from immunized mice. Finally, histochemistry analysis was performed to further visualize the necrosis in tumor tissues.

\section{Materials and methods}

Mice and cell lines. Male ICR mice, 3-4 weeks old (weighing 19-22 g), were purchased from Changzhou Cavens Experimental Animal Co. Ltd (Changzhou, Jiangsu, China). Mice were maintained at $20-26^{\circ} \mathrm{C}$ in pathogen-free conditions at a relative humidity of $40-65 \%$ in a 12:12 h light-dark cycle and fed ad libitum. All procedures in animal experiments were approved by the Animal Study Committee of Binzhou Medical University (Yantai, Shandong, China). H22 murine hepatocellular carcinoma cell line was purchased from The Type Culture Collection of the Chinese Academy of Sciences (Shanghai, China). Cells were cultured in RPMI-1640 (HyClone, GE Healthcare, Logan, UT, USA) supplemented with $10 \%$ fetal bovine serum (HyClone, GE Healthcare), $100 \mu \mathrm{g} / \mathrm{ml}$ streptomycin and $100 \mathrm{U} / \mathrm{ml}$ penicillin at $37^{\circ} \mathrm{C}$ with $5 \% \mathrm{CO}_{2}$.

Vaccine preparation. $\mathrm{H} 22$ cells were collected from the tissue culture flask and washed three times with PBS, then suspended in PBS so that each $1 \mathrm{ml}$ contained $1 \times 10^{7}$ $\mathrm{H} 22$ cells. A total of $100 \mu \mathrm{l}$ cell suspension was lysed by five cycles of freezing in $-20^{\circ} \mathrm{C}$ for $30 \mathrm{~min}$ and thawing in $37^{\circ} \mathrm{C}$ for $10 \mathrm{~min}$ and $100 \mu \mathrm{l}$ cell suspension was fixed with $0.025 \%$ glutaraldehyde at room temperature for $20 \mathrm{~min}$, subsequently fixed cells were washed three times with PBS. The H22 cell lysates and glutaraldehyde-fixed H22 cells were mixed with Freund's adjuvant (Sigma-Aldrich; Merck KGaA, Darmstadt, Germany) (1:1). The mixed method was as follows (14), H22 tumor cell lysate was mixed with Freund's adjuvant and a syringe was used to mix repeatedly and to form stable water-in-oil emulsion, which was named H22-TCL. Similarly, glutaraldehyde-fixed H22 cells were mixed with Freund's adjuvant using the same method to prepare the Fixed-H22-CELL vaccine.

Immunization protocols in tumor models. A total of three groups of 18 male ICR mice (in prophylactic, therapeutic or lung metastasis model, respectively) were randomly divided into three further groups of six animals each, which were treated with $100 \mu 1 \mathrm{PBS}, \mathrm{H} 22$-TCL or Fixed-H22-CELL, respectively. Vaccines were administrated using prophylactic or therapeutic strategies. For each immunization strategy, mice were subcutaneously immunized with separate vaccines or PBS in the left inguinal lymph node area.

In the prophylactic strategy, mice were immunized on days $-28,-21,-14$ and -7 . Subsequently, the tumor challenge experiment was performed by subcutaneously injecting $1 \times 10^{6} \mathrm{H} 22$ cells into the right flank on day 0 . Sera were collected weekly for immunoassay following initial immunization.
In the therapeutic strategy, mice were injected with $1 \times 10^{6}$ H22 cells into the right flank on day 0 and then immunized with separate vaccines on days 3,10 and 17. Tumor volume was evaluated every other day one week after the tumor challenge. The tumor volume was determined using the formula: Volume $=0.52 \mathrm{XY}^{2}$, where ' $\mathrm{X}$ ' is the larger diameter and ' $\mathrm{Y}$ ' is the smaller diameter. On day 21 , all mice in each group were sacrificed for tumor weight evaluation.

A further 18 male ICR mice were randomly divided into three groups of six animals in each, and mice were immunized on days $-28,-21,-14$ and -7 as described above, and then intravenously injected in the tail with $5 \times 10^{5} \mathrm{H} 22$ cells on day 0 to establish a lung metastasis model (15). On day 21 , all mice were sacrificed and the lungs were removed, followed by perfusion with 2-3 ml of Indian ink (Sangon Biotech Co., Ltd., Shanghai, China) using 22-gauge gavage needles and subsequently the excised lungs were fixed with $10 \%$ formalin for $36 \mathrm{~h}$ at room temperature. Lung metastasis was evaluated macroscopically by counting the metastatic nodules that were clearly visible on the lung surface. For microscopic observation, formalin-fixed lung tissues were stained with hematoxylin-eosin (H\&E) for $5 \mathrm{~h}$ at room temperature and the examined by light microscopy (magnification, x100).

ELISA analysis for serum anti-H22 antibody. A total of six mice of the three immunized groups in the prophylactic strategy were administered with separate vaccines every week for four consecutive weeks, and serum was collected every week following initial immunization. The anti-H22 antibodies present in the serum were evaluated using an ELISA, as described previously (16). Briefly, 96-well flat-bottomed ELISA plates were coated with $10 \mu \mathrm{g} /$ well of whole $\mathrm{H} 22$ cell lysates protein. Sera diluted at 1:50, and horseradish peroxidase-conjugated rabbit anti-mouse IgG (Beyotime Institute of Biotechnology, Haimen, China) diluted at $1: 100,000$ were used at $37^{\circ} \mathrm{C}$ for $2 \mathrm{~h}$. The enzyme reaction was developed using the peroxidase substrate $3,3^{\prime}, 5,5^{\prime}$-tetramethylbenzidine for $15-30 \mathrm{~min}$ at $37^{\circ} \mathrm{C}$, and quenched using $\mathrm{H}_{2} \mathrm{SO}_{4}(2 \mathrm{M})$ for $1 \mathrm{~min}$ at room temperature. The ELISA plate was read using a standard ELISA reader at $450 \mathrm{~nm}$. Each evaluation was performed in triplicate.

$T$ cell proliferation assay. Following one week after the final immunization of the prophylactic strategy, splenocytes were isolated from sacrificed mice of each immunized and PBS group. Firstly, spleens were ground and passed through a $200 \mu \mathrm{m}$ filter under sterile conditions. Erythrocytes were lysed at room temperature using Tris- $\mathrm{NH}_{4} \mathrm{Cl}$ for $5 \mathrm{~min}(\mathrm{pH}$ 7.2). The splenocytes were washed three times with PBS and resuspended in RMPI-1640 supplemented with 10\% FBS. The spleen cells $\left(2 \times 10^{5}\right.$ cells/well $)$ were incubated in triplicate in 96-well plates for $72 \mathrm{~h}$ in the presence or absence of $\mathrm{H} 22$ cell lysate $(100 \mu \mathrm{g} / \mathrm{ml})$ at $37^{\circ} \mathrm{C}$. ConA $(5 \mu \mathrm{g} / \mathrm{ml})$ was used as a positive control. Cell proliferation was analyzed by MTT assay. Following a 72-h incubation, the supernatant in each well was discarded and $10 \mu \mathrm{l}$ MTT $(5 \mathrm{mg} / \mathrm{ml})$ was added to each well. Following incubation at $37^{\circ} \mathrm{C}$ for an additional $4 \mathrm{~h}, 100 \mu \mathrm{l}$ dimethyl sulfoxide was pipetted to solubilize the product for $10 \mathrm{~min}$ at room temperature. Subsequently, the 96 -well plate was evaluated using an ELISA reader at $570 \mathrm{~nm}$. All assays were performed in triplicate. 

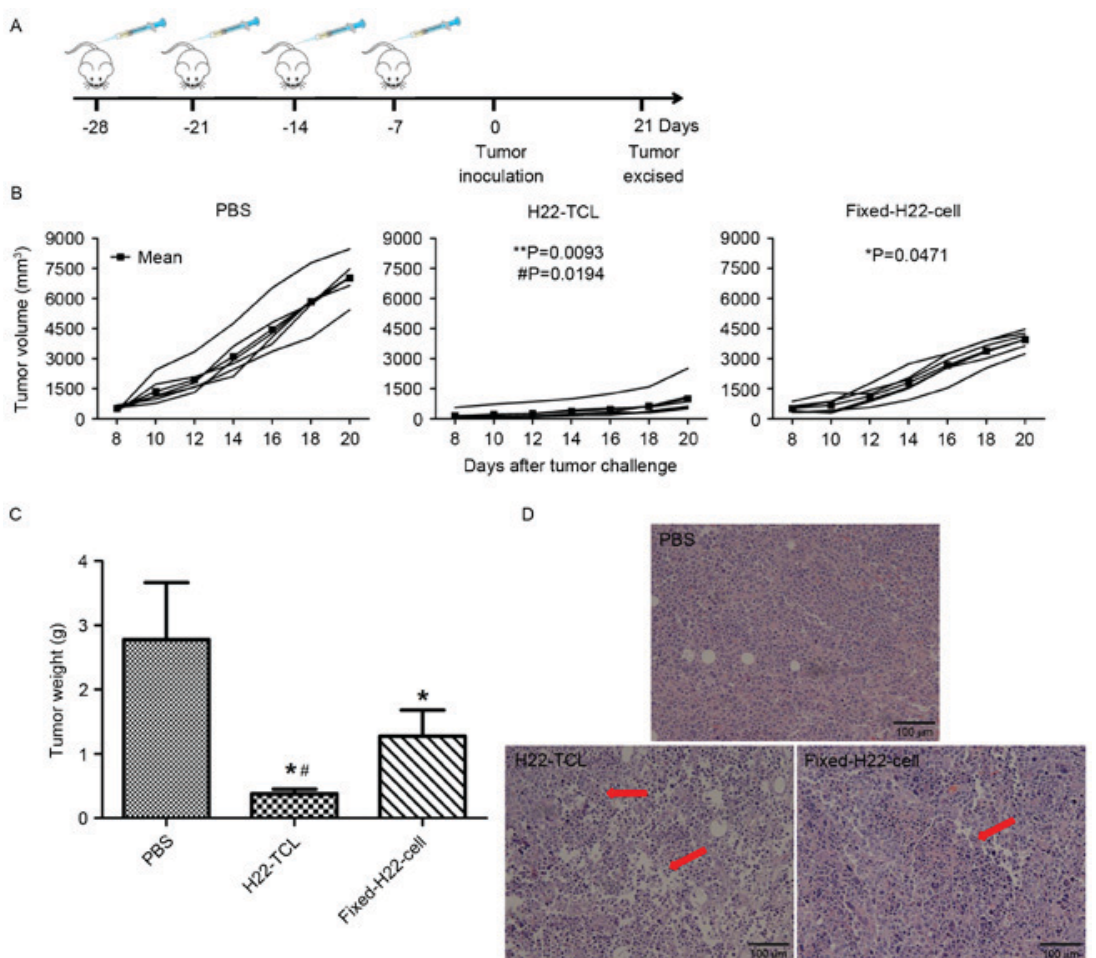

Figure 1. Prophylactic antitumor effect induced by various vaccines in subcutaneous H22 hepatocellular carcinoma-bearing mice. Male ICR mice (n=6 per group) were inoculated subcutaneously with PBS, H22-TCL or Fixed-H22-CELL on days $-28,-21,-14$ and -7. On day 0, 1x10 ${ }^{6}$ H22 cells were injected subcutaneously for tumor challenge. All mice were sacrificed and tumors were excised on day 21. (A) Schematic diagram of the immunization procedure. (B) In vivo evaluation of tumor growth, each line represents a single mouse. Tumor volume was determined using calipers every other day. (C) Weight of tumor from mice immunized with PBS, H22-TCL or Fixed-H22-CELL. (D) Histopathological observation of excised tumor tissues (hematoxylin and eosin staining; magnification, $\mathrm{x} 200)$. Tumor necrosis areas are indicated with an arrow. ${ }^{*} \mathrm{P}<0.05$ vs. PBS; ${ }^{* *} \mathrm{P}<0.01$ vs. PBS. ${ }^{\#} \mathrm{P}<0.05$ vs. Fixed-H22-CELL.

Histological evaluation of the subcutaneous tumor tissues. Subcutaneous tumor tissues were fixed with $10 \%$ formalin, embedded in paraffin and cut into $4 \mu \mathrm{m}$ sections. H\&E staining was then performed on tissues sections for $5 \mathrm{~h}$ at room temperature. All tissue sections were evaluated by light microscopy, at magnification, x100 for histological changes that may be associated with the treatment.

Toxicity assessment. The treatment-associated toxicity was mainly evaluated by analyzing the mice weight alterations. The tissues (heart, liver, spleen, lung and kidney) were then fixed with $10 \%$ formalin and embedded in paraffin. The $4 \mu \mathrm{m}$ tissue sections were stained with $\mathrm{H} \& \mathrm{E}$ for $5 \mathrm{~h}$ at room temperature, and evaluated by light microscopy, at magnification, x100.

Statistical analysis. Data are presented as the mean \pm standard deviation. Multiple comparisons were analyzed using one-way analysis of variance with Tukey's post hoc test. $\mathrm{P}<0.05$ was considered to indicate a statistically significant difference. Analysis was performed in GraphPad Prism 6 (GraphPad Software, La Jolla, CA, USA).

\section{Results}

H22-TCL vaccination induces prophylactic anti-hepatocellular carcinoma effects. A vaccination protocol was designed as shown in Fig. 1A to analyze the prophylactic anti-hepatocellular carcinoma effect. As presented in Fig. 1B, PBS-treated mice developed rapidly progressive disease following tumor inoculation. Conversely, H22-bearing mice treated with Fixed-H22-CELL or H22-TCL exhibited slower tumor growth, and the inhibition effect of H22-TCL was the most significant. The excised tumor weight revealed similar results, although the groups of mice immunized with Fixed-H22-CELL and H22-TCL exhibited a significantly lower mean tumor weight compared with the PBS group (Fig. $1 \mathrm{C} ; \mathrm{P}<0.05$ ). The tumor weight of mice immunized with H22-TCL was significantly decreased compared with mice treated with Fixed-H22-CELL (Fig. 1C; $\mathrm{P}<0.05$ ). The sections of excised tumors were stained with $H \& E$ and viewed using a microscope, and the tumor sections from the H22-TCL and Fixed-H22-CELL groups were detected with degeneration necrosis in tumor cells and the tumor cell nucleus were dissolved. Conversely, these phenomena were not present in tumor tissue sections from PBS-treated mice (Fig. 1D).

H22-TCL vaccination induces therapeutic anti-hepatocellular carcinoma effects. More relevant to the treatment of tumor is the therapeutic potential, thus, a therapeutic vaccination protocol was designed as shown in Fig. 2A. The results in the therapeutic strategy were in accordance with that of prophylactic strategy. In comparison with the PBS-treated group, tumor growth was significantly inhibited in mice immunized with H22-TCL or Fixed-H22-CELL (Fig. 2B). Furthermore, the tumor growth of the H22-TCL group was significantly slower compared with the Fixed-H22-CELL group $(\mathrm{P}<0.05)$. The excised tumor weight revealed similar results, as the group of mice immunized with H22-TCL 
A
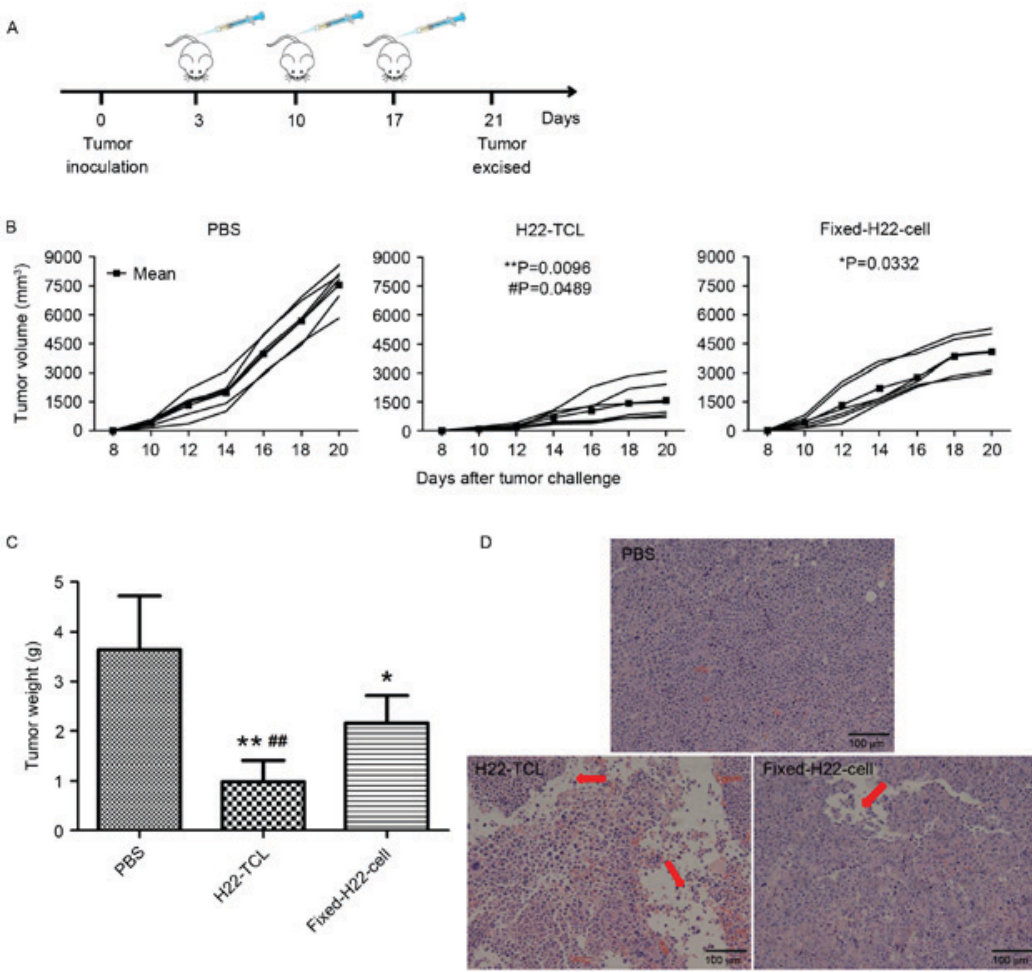

Figure 2. Therapeutic antitumor effect induced by various vaccines in subcutaneous $\mathrm{H} 22$ hepatocellular carcinoma-bearing mice. Male ICR mice (n=6 per group) were injected subcutaneously with $1 \times 10^{6} \mathrm{H} 22$ cells on day 0 , and injected with PBS, H22-TCL or Fixed-H22-CELL on days 3,10 and 17 . Mice were sacrificed and tumors were excised on day 21. (A) Schematic diagram of the immunization procedure. (B) In vivo evaluation of tumor growth, each line represents a single mouse. Tumor volume was determined using calipers every other day. (C) Weight of tumors from mice immunized with PBS, H22-TCL or Fixed-H22-CELL. (D) Histopathological observation of excised tumor tissues (hematoxylin and eosin staining; magnification, x200). Tumor necrosis areas are indicated by arrows. ${ }^{*} \mathrm{P}<0.05$ vs. $\mathrm{PBS} ;{ }^{* *} \mathrm{P}<0.01$ vs. PBS. ${ }^{*} \mathrm{P}<0.05$ vs. Fixed-H22-CELL; ${ }^{\# \#} \mathrm{P}<0.01$ vs. Fixed-H22-CELL.

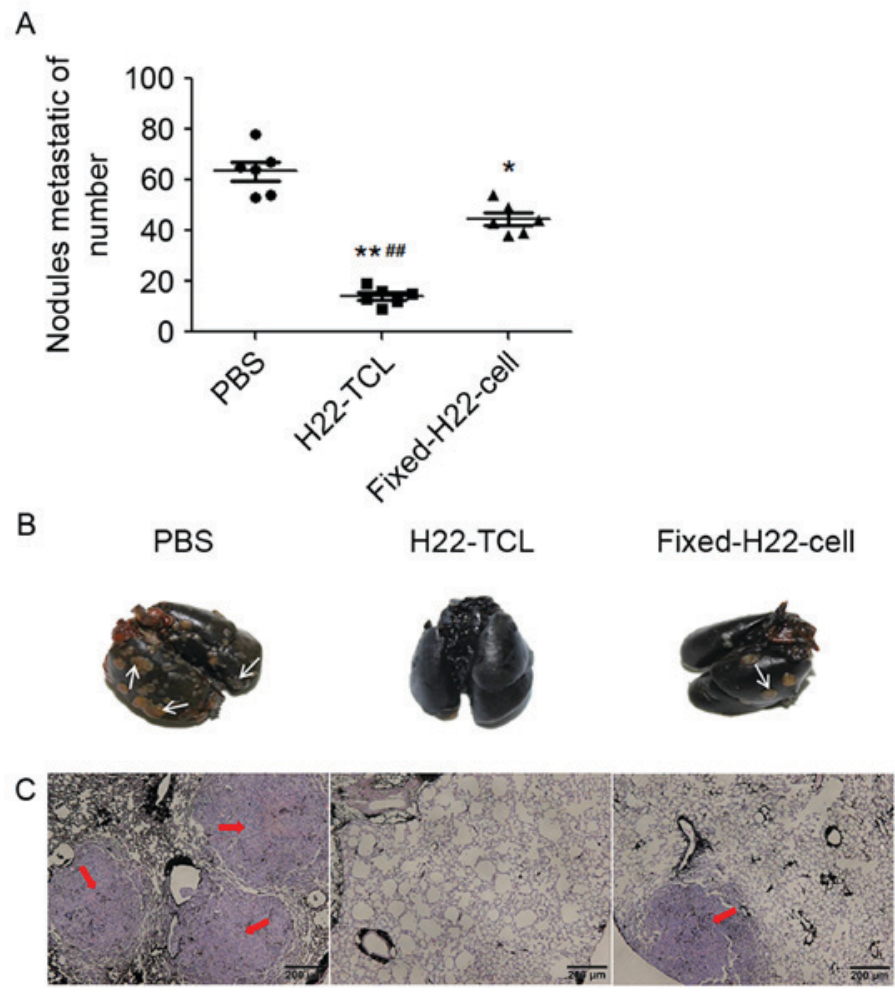

Figure 3. Prophylactic inhibition on tumor metastasis induced by various vaccines. Male ICR mice ( $\mathrm{n}=6$ per group) administrated with PBS, H22-TCL or Fixed-H22-CELL on days $-28,-21,-14$ and -7 . On day $0,5 \times 10^{5} \mathrm{H} 22$ cells were injected intravenously for tumor challenge. On day 21 , the mice were sacrificed and lung metastases were visualized by staining the lung parenchyma with India ink. (A) Final number of macroscopic lung metastatic nodules. (B) Macroscopic appearance of representative murine lungs, staining with India ink. Normal lung parenchyma was stained black and white metastatic nodules were clearly visible, tumor areas are indicated by white arrows. (C) Microscopic appearance of representative murine lungs, tumor areas are indicated by red arrows (magnification, $\mathrm{x} 100$ ). ${ }^{*} \mathrm{P}<0.05$ vs. PBS; ${ }^{* *} \mathrm{P}<0.01$ vs. PBS. ${ }^{\#} \mathrm{P}<0.05$ vs. Fixed-H22-CELL; ${ }^{\# \#} \mathrm{P}<0.01$ vs. Fixed-H22-CELL. 
A

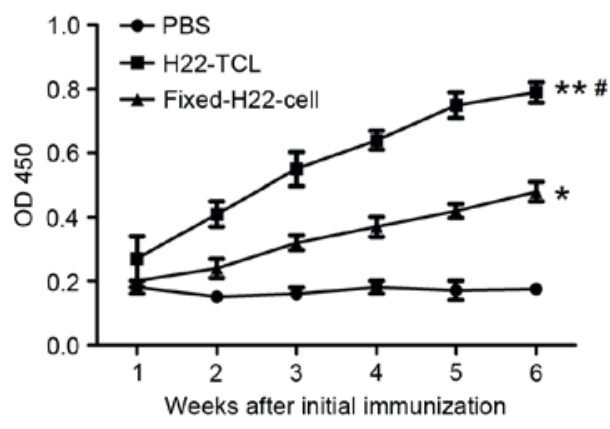

B

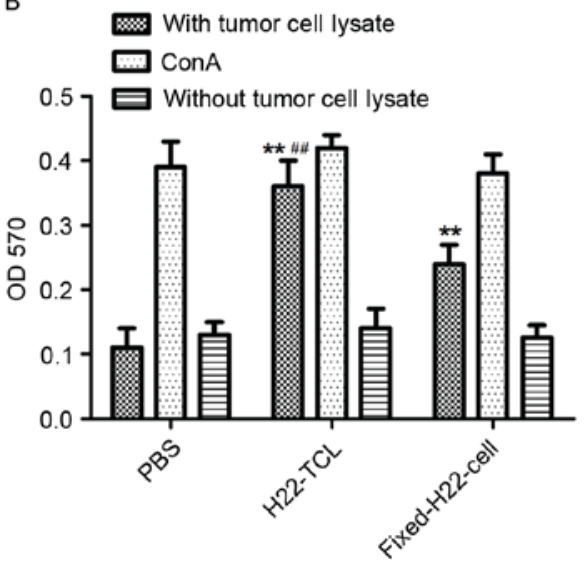

Figure 4. Immune responses elicited by various vaccines. Male ICR mice ( $\mathrm{n}=6$ per group) were immunized with PBS, H22-TCL or Fixed-H22-CELL every week for 4 consecutive weeks. Blood was collected from each mouse every week after the initial injection to obtain sera for immunoassay. Following one week after the last immunization, the spleens were removed and T lymphocytes proliferative responses were assayed with $\mathrm{H} 22$ lysate in vitro. (A) Detection of specific anti-H22 antibodies. (B) Effect of each vaccination on T lymphocytes proliferation. RPMI-1640 media and ConA were used as the negative and positive controls, respectively. ${ }^{*} \mathrm{P}<0.05$ vs. PBS; ${ }^{* *} \mathrm{P}<0.01$ vs. PBS. "P $<0.05$ vs. Fixed-H22-CELL; "\# $\mathrm{P}<0.01$ vs. sp16 Fixed-H22-CELL. OD, optical density.

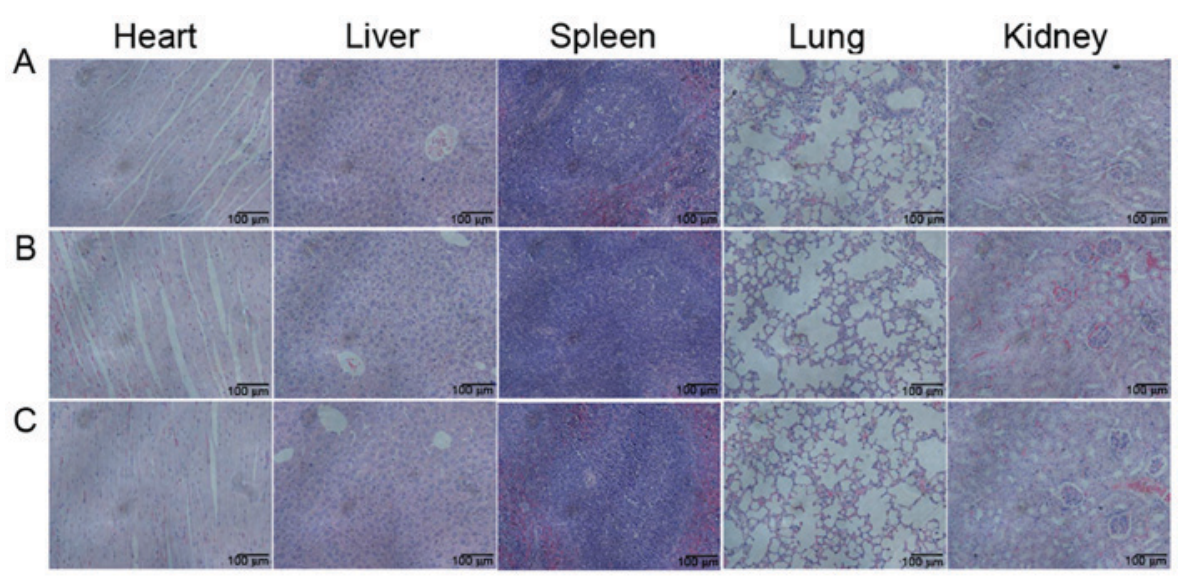

Figure 5. General toxicity observation. Hematoxylin and eosin staining (magnification, x200) of the heart, liver, spleen, lung and kidney. (A) PBS, (B) H22-TCL and (C) Fixed-H22-CELL vaccines.

exhibited the lowest mean tumor weight among the three groups (Fig. 2C). H\&E staining of the excised tumor tissues from the H22-TCL group further indicated that H22-TCL immunization induced the largest areas of inflammatory infiltrates and continuous degenerative necrosis among the three experimental groups (Fig. 2D).

H22-TCL vaccination induces prophylactic anti-metastasis effects. Subsequently, the present study investigated whether the two vaccines could inhibit the growth of pulmonary metastasis. The results are presented in Fig. 3. In comparison with the PBS-treated mice, the number of metastatic nodules following treatment with H22-TCL or Fixed-H22-CELL was significantly reduced (Fig. $3 \mathrm{~A} ; \mathrm{P}<0.01$ and $\mathrm{P}<0.05$, respectively compared with the PBS group), and the number of macroscopic metastatic nodules in the H22-TCL group was the lowest $(\mathrm{P}<0.01$ compared with the Fixed-H22-CELL group). Furthermore, microscopic evaluation supported the macroscopic findings, as the number of microscopic metastatic foci in the H22-TCL group was the lowest (Fig. 3B and C).
H22-TCL vaccination induces a high level of anti-H22 antibody production. In order to investigate the humoral immune responses elicited by various vaccines, ELISA was performed to determine the expression level of anti-H22 antibodies in sera collected from immunized mice. Compared with the PBS-treated group, H22 specific IgG antibody responses were more evident in mice immunized with H22-TCL or Fixed-H22-CELL (Fig 4A; $\mathrm{P}<0.01$ and $\mathrm{P}<0.05$, respectively). In addition, the antibody expression level of the H22-TCL group was significantly increased compared with that of the Fixed-H22-CELL group $(\mathrm{P}<0.05)$.

H22-TCL vaccination induces a high T lymphocyte proliferation activity. An MTT assay was performed to determine the proliferation ability of lymphocytes from immunized mice. As presented in Fig. 4B, compared with the PBS group, a significant increase in the proliferation of lymphocytes in the H22-TCL and Fixed-H22-CELL treatment groups was revealed $(\mathrm{P}<0.01)$. In addition, the proliferation activity of lymphocytes from mice immunized with H22-TCL was 
significantly increased compared with that from mice immunized with Fixed-H22-CELL ( $\mathrm{P}<0.01)$.

Toxicity observation. All mice appeared generally healthy, without any noteworthy changes in appearance, fur, habits and body weight following vaccine immunization. The effects of the vaccines on normal tissues (heart, liver, spleen, lung and kidney) were further examined. No pathologic changes were observed in the organs of the immunized mice macroscopically (Fig. 5). Microscopic examination also revealed that H22-TCL and Fixed-H22-CELL vaccines induced no damage in the organs excised from immunized mice.

\section{Discussion}

Hepatocellular carcinoma is reported to be the second leading cause of cancer-associated mortality worldwide, and its incidence is rising (17). However, notwithstanding great advances, no systemic chemotherapeutic protocol has proved to be successful in HCC treatment (18). Along with the development of immunology and further understanding of the mechanisms of tumorigenesis, tumor cell vaccines have become a new cancer treatment research. Compared with traditional therapies, tumor cell vaccines have the characteristic of strong specificity, broad antitumor spectrum, low tolerance and have obtained success in clinical trials $(19,20)$.

Although cancer cell vaccines have been studied as a promising cancer treatment strategy for decades, the antigen form, which is the most effective in cancer vaccine preparation, has not yet been clearly demonstrated. The antitumor efficiency of vaccines prepared by tumor cell lysate and glutaraldehyde-fixed tumor cells, two commonly used antigen forms (21-23), was evaluated in the present study. The results of the present study demonstrated that the tumor cell lysate-based vaccine induced increased significant inhibition on tumor growth and metastasis compared with the glutaraldehyde-fixed tumor cell-based vaccine, which may be the result of the more evident antigen-specific humoral and cellular immune responses. These results implied that whole tumor cell lysate may be a more effective antigen form in cancer vaccine preparation compared with glutaraldehyde-fixed tumor cells, which would have clinical significance for cancer vaccine preparation.

Recurrence and metastasis are typically the causes of failure of multidisciplinary treatment for patients with $\operatorname{HCC}(24,25)$. The present study evaluated the anti-metastasis efficacy of the two vaccines using the tail venous injection lung metastasis model. Consistent with the results of the subcutaneous tumor model, the inhibition of metastasis by tumor cell lysate-based vaccine was increased compared with the glutaraldehyde-fixed tumor cell-based vaccine (Fig. 3). Microscopic evaluation of lung tissues further demonstrated that the number of microscopic metastatic foci in the tumor cell lysate-based vaccine group was lowest. All the results revealed that the tumor cell lysate-based vaccine may be used to decrease residual or metastatic tumor cells for patients with HCC.

Taken together, the results from the present study suggested that whole tumor cell lysate immunization may evoke a stronger immune response compared with glutaraldehyde-fixed tumor cells, which may result in a more significant inhibition on $\mathrm{H} 22$ hepatocellular carcinoma growth and metastasis. Collectively, these results indicated that whole tumor cell lysate may be a more effective antigen form in cancer cell vaccine preparation. The findings of the present study may provide a rationale for the further optimization of cancer cell vaccines and prompt further studies.

\section{Acknowledgements}

The present study was supported by the National Natural Science Foundation of China (grant no. 81541158), Promotive Research Fund for Young and Middle-aged Scientists of Shandong Province (grant no. BS2014YY051), Shandong Provincial Natural Science Foundation (grant no. ZR2015PH002), Medicine and Health Science Technology Plan of Shandon Province (grant no. 2014WS0479) and A Project of Shandong Province Higher Educational Science and Technology Program (grant no. J15LM51).

\section{References}

1. Ray K: Liver cancer: The promise of new approaches in the management of hepatocellular carcinoma-adding to the toolbox? Nat Rev Gastroenterol Hepatol 10: 195, 2013.

2. Dong B, Dai G, Xu L, Zhang Y, Ling L, Sun L and Lv J: Tumor cell lysate induces the immunosuppression and apoptosis of mouse immunocytes. Mol Med Rep 10: 2827-2834, 2014.

3. Grotz TE, Kottschade L, Pavey ES, Markovic SN and Jakub JW: Adjuvant GM-CSF improves survival in high-risk stage iiic melanoma: A single-center Study. Am J Clin Oncol 37: 467-472, 2014.

4. Tada F, Abe M, Hirooka M, Ikeda Y, Hiasa Y, Lee Y, Jung NC, Lee WB, Lee HS, Bae YS and Onji M: Phase I/II study of immunotherapy using tumor antigen-pulsed dendritic cells in patients with hepatocellular carcinoma. Int J Oncol 41: 1601-1609, 2012.

5. Chen IJ, Yen CF, Lin KJ, Lee CL, Soong YK, Lai CH and Lin CT: Vaccination with OK-432 followed by TC-1 tumor lysate leads to significant antitumor effects. Reprod Sci 18: 687-694, 2011.

6. Huang L and Ohno T: Protective antitumor immunity induced by fixed tumor cells in combination with adjuvant in a murine hepatoma model. Cancer Lett 202: 153-159, 2003.

7. Novaković S, Stegel V, Kopitar A, Ihan A and Novaković BJ: Preventive and therapeutic antitumor effect of tumor vaccine composed of $\mathrm{CpG}$ ODN class $\mathrm{C}$ and irradiated tumor cells is triggered through the APCs and activation of CTLs. Vaccine 25: 8241-8256, 2007.

8. Suckow MA, Rosen ED, Wolter WR, Sailes V, Jeffrey R and Tenniswood M: Prevention of human PC-346C prostate cancer growth in mice by a xenogeneic tissue vaccine. Cancer Immunol Immunother 56: 1275-1283, 2007.

9. Yin W, He Q, Hu Z, Chen Z, Qifeng M, Zhichun S, Zhihui Q, Xiaoxia N, Li J and Gao J: A novel therapeutic vaccine of GM-CSF/TNFalpha surface-modified RM-1 cells against the orthotopic prostatic cancer. Vaccine 28: 4937-4944, 2010.

10. Wang ZY, Xing Y, Liu B, Lu L, Huang X, Ge CY, Yao WJ, $\mathrm{Xu}$ ML, Gao ZQ, Cao RY, et al: Protective antitumor immunity induced by tumor cell lysates conjugated with diphtheria toxin and adjuvant epitope in mouse breast tumor models. Chin J Cancer 31: 295-305, 2012.

11. Xu M, Zhou L, Zhang P, Lu Y, Ge C, Yao W, Xing Y, Xiao W, Dong Y, Wu J, et al: Enhanced antitumor efficacy by combination treatment with a human umbilical vein endothelial cell vaccine and a tumor cell lysate-based vaccine. Tumour Biol 34: 3173-3182, 2013.

12. Copier J and Dalgleish A: Whole-cell vaccines: A failure or a success waiting to happen? Curr Opin Mol Ther 12: 14-20, 2010.

13. Maki RG, Livingston PO, Lewis JJ, Janetzki S, Klimstra D, Desantis D, Srivastava PK and Brennan MF: A phase I pilot study of autologous heat shock protein vaccine HSPPC-96 in patients with resected pancreatic adenocarcinoma. Dig Dis Sci 52: 1964-1972, 2007. 
14. Fernandez P, Petres S, Mécheri S, Gysin J and Scherf A: Strain-transcendent immune response to recombinant Var2CSA DBL5- $\varepsilon$ domain block P. falciparum adhesion to placenta-derived BeWo cells under flow conditions. PLoS One 5: e12558, 2010.

15. Blezinger P, Wang J, Gondo M, Quezada A, Mehrens D, French M, Singhal A, Sullivan S, Rolland A, Ralston R and Min W: Systemic inhibition of tumor growth and tumor metastases by intramuscular administration of the endostatin gene. Nat Biotechnol 17: 343-348, 1999.

16. Xu M, Xing Y, Zhou L, Yang X, Yao W, Xiao W, Ge C, Ma Y Yang J, Wu J, et al: Improved efficacy of therapeutic vaccination with viable human umbilical vein endothelial cells against murine melanoma by introduction of OK432 as adjuvant. Tumour Biol 34: 1399-1408, 2013.

17. Fowlkes V, Wilson CG, Carver W and Goldsmith EC: Mechanical loading promotes mast cell degranulation via RGD-integrin dependent pathways. J Biomech 46: 788-795, 2013.

18. Ozturk M and Oter S: Molecular approach to treatment of hepatocellular carcinoma: New hope for therapeutic targets. J Exp Integr Med 1: 83-84, 2011.

19. Couzin-Frankel J: Breakthrough of the year 2013. Cancer immunotherapy. Science 342: 1432-1433, 2013.
20. Elert E: Calling cells to arms. Nature 504: S2-S3, 2013.

21. Yuan X, Li W, Cui Y, Zhan Q, Zhang C, Yang Z, Li X, Li S, Guan Q and Sun X: Specific cellular immune response elicited by the necrotic tumor cell-stimulated macrophages. Int Immunopharmacol 27: 171-176, 2015.

22. Kawahara $\mathbf{M}$ and Takaku H: Intradermal immunization with combined baculovirus and tumor cell lysate induces effective antitumor immunity in mice. Int J Oncol 43: 2023-2030, 2013.

23. He A, Zhang W, Xu K, Wang J, Yang Y and Chao X: Anti-tumor immune responses in immune-reconstituted mice injected with a tumor vaccine. Med Oncol 29: 2261-2269, 2012.

24. Ishii Y, Sakamoto T, Ito R and Yanaga K: Anti-angiogenic therapy on hepatocellular carcinoma development and progression. J Surg Res 158: 69-76, 2010.

25. Liang Y and Sun H: The tumor protection effect of high-frequency administration of whole tumor cell vaccine and enhanced efficacy by the protein component from Agrocybe aegerita. Int J Clin Exp Med 8: 6914-6925, 2015. 